\title{
Nascent Regional System for Alerting Infection Preventionists about Patients with Multidrug-Resistant Gram-Negative Bacteria: Implementation and Initial Results
}

\author{
Marc B. Rosenman, MD; ${ }^{1,2}$ Kinga A. Szucs, MD; ${ }^{1}$ S. Maria E. Finnell, MD, MS; ${ }^{1,2}$ Shahid Khokhar, BA; ${ }^{2}$ James Egg, BS; ${ }^{2}$
} Larry Lemmon, BS; $;^{2}$ avid C. Shepherd, DO, MBA; ${ }^{3}$ Jeff Friedlin, DO; Xiaochun Li, PhD; ${ }^{1,4}$ Abel N. Kho, MD, MS ${ }^{2,5}$

\begin{abstract}
овJECTIVE. To build and to begin evaluating a regional automated system to notify infection preventionists (IPs) when a patient with a history of gram-negative rod multidrug-resistant organism (GNRMDRO) is admitted to an emergency department (ED) or inpatient setting.
\end{abstract}

DESIGN. Observational, retrospective study.

SETTING. Twenty-seven hospitals, mostly in the Indianapolis metropolitan area, in a health information exchange (HIE).

PATIENTS. During testing of the new system: 80,180 patients with microbiology cultures between October 1, 2013, and December 31 , 2013; 573 had a GNRMDRO.

Methods/intervention. A Health Level Seven (HL7) data feed from the HIE was obtained, corrected, enhanced, and used for decision support (secure e-mail notification to the IPs). Retrospective analysis of patients with microbiology data (October 1, 2013, through December 31, 2013) and subsequent healthcare encounters (through February 6, 2014).

RESULTS. The 573 patients (median age, 66 years; $68 \%$ women) had extended-spectrum $\beta$-lactamase-producing Enterobacteriaceae ( $78 \%$ ), carbapenem-resistant Enterobacteriaceae (7\%), Pseudomonas aeruginosa (9\%), Acinetobacter baumannii (3\%), or other GNR (3\%). Body sources were urine $(68 \%)$, sputum/trachea/bronchoalveolar lavage $(13 \%)$, wound/skin $(6 \%)$, blood $(6 \%)$, or other/unidentified $(7 \%)$. Between October 1, 2013, and February 6, 2014, 252 (44\%) of 573 had an ED or inpatient encounter after the GNRMDRO culture, 47 (19\% of 252) at an institution different from where the culture was drawn. During the first 7 weeks of actual alerts (January 29,2014 , through March 19, 2014), alerts were generated regarding 67 patients (19 of 67 admitted elsewhere from where the culture was drawn).

CONCLUSIONS. It proved challenging but ultimately feasible to create a regional microbiology-based alert system. Even in a few months, we observed substantial crossover between institutions. This system, if it contributes to timely isolation, may help reduce the spread of GNRMDROs.

Infect Control Hosp Epidemiol 2014;35(S3):S40-\$47

Recent outbreaks and deaths caused by multidrug-resistant gram-negative organisms among hospitalized patients in the United States have underscored the danger posed by these infections.' The Centers for Disease Control and Prevention has encouraged healthcare departments and providers to "act aggressively to prevent the emergence and spread" of carbapenem-resistant Enterobacteriaceae (CRE) ${ }^{2,3}$ The prevalence of multidrug-resistant Klebsiella pneumonia and Escherichia coli in the United States increased from $7 \%$ in 2000 to $13 \%$ in $2008 .{ }^{4}$ In France, the incidence of extended-spectrum $\beta$-lactamase-producing Enterobacteriaceae (ESBL-E) has climbed alarmingly (from 0.17 to 0.48 per 1,000 patient- days). ${ }^{5}$ In Hungary, rates of multidrug-resistant E. coli, Klebsiella, and Acinetobacter have increased markedly. ${ }^{6}$ In Pakistan, ${ }^{7}$ India, ${ }^{8}$ and many other nations, ${ }^{9}$ reports from hospitals document rapid increases in gram-negative rod multidrugresistant organism (GNRMDRO) infections.

A minority of GNRMDROs arise in the outpatient setting, and many of the colonized patients have no healthcareassociated risk factors and can bring these bacteria into hospitals on admission. ${ }^{10}$ After discharge from a university hospital, median time to clearance of ESBL-E (based on rectal screening) was 6.6 months; the authors recommended "screening for ESBL-E and contact isolation precautions at

Affiliations: 1. Indiana University School of Medicine, Indianapolis, Indiana; 2. Regenstrief Institute, Indianapolis, Indiana; 3. Shepherd Internal Medicine, Indianapolis, Indiana; 4. Indiana University School of Public Health, Indianapolis, Indiana; 5. Feinberg School of Medicine, Northwestern University, Chicago, Illinois.

Received May 27, 2014; accepted June 3, 2014; electronically published September 15, 2014.

(C) 2014 by The Society for Healthcare Epidemiology of America. All rights reserved. 0899-823X/2014/35S3-0007\$15.00. DOI: 10.1086/677833 
hospital readmission ... for all patients identified as testing positive for ESBL-E infection during an earlier hospital stay." ${ }^{\prime 1}$ Substantial rates of interhospital spread of Clostridium difficile $^{12}$ and methicillin-resistant Staphylococcus aureus (MRSA) $)^{13}$ have been documented in Orange County, California. The authors modeled MRSA surveillance and contact isolation practices across all Orange County hospitals and illustrated the interconnectedness of the system ${ }^{14}$-improved coordination could reduce the spread of MDROs synergistically. ${ }^{14,15}$

Kho et $\mathrm{al}^{16}$ found, in the original 5 hospital systems in the Indiana Network for Patient Care (INPC), that in $10 \%$ of admissions the admitting hospital was not aware of the patient's previous history of MRSA or vancomycin-resistant enterococci (VRE) at a different institution, and contact isolation was frequently indicated but not ordered. The regional alert system for MRSA and VRE generated e-mail alerts to infection preventionists (IPs) for patients admitted to an emergency department (ED) or inpatient setting; approximately $20 \%$ were based on data from a hospital system different from where the patient was being admitted. ${ }^{17}$ This MRSA and VRE alert system relied on the IPs themselves for data entry.

In light of the growing danger of GNRMDRO outbreaks caused by patients who carry these organisms into the hospital and the potential of the prior system to reduce spread, we constructed a new GNRMDRO alert system that builds its registry from microbiology culture data. We designed the new system to draw data from microbiology cultures for several reasons: (1) to remove the IPs' manual data-entry burden, (2) to allow flexibility in the selection of bacteria for alerts, (3) to enable more efficient analysis of microbiology data, and (4) to expand the alerts geographically by including more hospitals' microbiology data and more ED and inpatient encounters.

\section{METHODS}

\section{Overview}

The development of the Regional Electronic Infection Control Network (REICON) for GNRMDRO is described more fully in Advances in the Prevention and Control of HealthcareAssociated Infections. ${ }^{18}$ REICON parses microbiology culture data from 27 hospitals and stores these data as a GNRMDRO registry; it then sends e-mail alerts to notify IPs when a patient with a history of GNRMDRO is newly admitted anywhere in the system. The REICON microbiology data parser began processing culture data on October 1, 2013, and the e-mail alerts were initiated later (as described below). This study was approved by the Institutional Review Board of Indiana University.

\section{Indiana Network for Patient Care}

The INPC is a leading operational regional health information exchange (HIE), formed in 1994 by the Regenstrief Institute and the 5 major hospital systems in Indianapolis. ${ }^{19}$ Its primary purpose is clinical data exchange to improve the quality and efficiency of health care; a secondary purpose is research. ${ }^{20}$ Since 2009, the number of hospital systems has expanded to more than 25 . We selected the top 12 hospital systems on the basis of microbiology message volume, and the 27 hospitals in those systems range in size from 16 to 1,510 beds (mean, 334; standard deviation, 370). Seventeen of 27 are in urban or suburban areas; 10 are in smaller towns. There are 2 children's hospitals. This population encompasses 1.9 million patients (age: $35 \% 24$ years or younger, $12 \% 65$ years or older; race: $80 \%$ white, $15 \%$ black; ethnicity: $6 \%$ Hispanic).

\section{Previous MRSA/VRE Registry and Alert System}

In the MRSA/VRE registry and alert system, ${ }^{16,17,21,22}$ variables such as culture site, infection status, study action status, barrier isolation status, and infection control comments were embedded in the INPC for IPs to enter patient information. Then, whenever a patient was admitted to a hospital or ED, the INPC's receipt of the admission, discharge, and transfer (ADT) Health Level Seven version 2 (HL7v2) message initiated an automated process that checked the MRSA/VRE registry; if positive, an e-mail alert was sent to the IP at the admitting hospital.

\section{New Microbiology Culture HL7 Correction Engine (the "REICON Transform")}

A significant barrier to the development of REICON was that microbiology data sent by hospitals into the HIE are often not structured in standard observation result (ORU) HL7v2 format. HL7v2 is an international standard for sending healthcare data messages; it is designed to facilitate the "interoperability" of systems and institutions ${ }^{23}$ but is difficult to use for multilayered results like microbiology cultures. As a result, microbiology data in an HIE, while useful for clinicians looking up patients one by one, are usually not a substrate suitably structured for sending e-mail alerts to IPs.

Additional HL7v2 processing by REICON made the microbiology culture data more useful. The REICON microbiology HL7v2 correction engine deals with incorrect HL7 message structure and/or content in the inbound ORU messages. It parses the 6 key data types and elements needed for infection control alerts: the organism, the antibiotics tested, the minimum inhibitory concentrations of the antibiotics, the susceptibility interpretation for each antibiotic, the body source from which the patient's culture was drawn, and the healthcare facility where it was drawn. It also looks for evidence that an assay for ESBL or CRE was positive. These parsed data elements get stored in the REICON schema in Oracle and, as structured data, can subsequently be integrated into e-mail alerts to IPs whenever a patient with a history of GNRMDRO is admitted to a hospital or ED. 
TABLE 1. Five Rules for Labeling a Bacterium as a Gram-Negative Rod Multidrug-Resistant Organism in the Regional Electronic Infection Control Network

\begin{tabular}{|c|c|c|}
\hline Rule & Organism category $^{a}$ & Definition \\
\hline 1 & Enterobacteriaceae & Confirmed production of an extended-spectrum $\beta$-lactamase \\
\hline 2 & Enterobacteriaceae & Confirmed carbapenemase production \\
\hline 3 & Pseudomonas aeruginosa & $\begin{array}{l}\text { Resistant to } 3 \text { or more classes of the following: } \\
\text { - Aminoglycosides (gentamicin, tobramycin, or amikacin) } \\
\text { - Fluoroquinolones (ciprofloxacin or levofloxacin) } \\
\text { - Carbapenems (imipenem or meropenem) } \\
\text { - } \beta \text {-Lactams (piperacillin/tazobactam, or \{ceftazidime and cefepime }\} \text { ) }\end{array}$ \\
\hline 4 & Acinetobacter baumannii & $\begin{array}{l}\text { Resistant to } 3 \text { or more classes of the following: } \\
\text { - Aminoglycosides (gentamicin, tobramycin, or amikacin) } \\
\text { - Fluoroquinolones (ciprofloxacin or levofloxacin) } \\
\text { - Carbapenems (imipenem or meropenem) } \\
\text { - } \beta \text {-Lactams (ampicillin/sulbactam, or \{ceftazidime and cefepime\}) }\end{array}$ \\
\hline 5 & Other gram-negative bacterium not listed above & Resistant to all antibiotics tested, excluding colistin or tigecycline ${ }^{b}$ \\
\hline
\end{tabular}

"For the list of organisms in each category, please contact the authors.

b The rationale for this definition is that colistin and tigecycline are antibiotics of last resort (and are not always part of the laboratory susceptibility assay). At least one of the large hospital systems uses this definition because it at least wants to be aware of the presence of any organism resistant to all other antibiotics tested.

\section{A Dictionary Term for Gram-Negative Superbug}

When the REICON engine structures the microbiology data, it also evaluates that content against criteria, developed in consultation with the IPs, for 5 categories of GNRMDRO (Table 1). If the criteria are met, an additional data element (GNR_MDRO) is written into the REICON database. GNR_MDRO stores which of the 5 "rules" was met and which version of the rules was applied. Our approach allows these rules to be rewritten, to deal with new or evolving superbug criteria in future years.

\section{ADT Hospitalization Messages and Master Patient Index Lookup}

The patient identifiers in inbound ADT messages for $\mathrm{ED}$ visits and hospitalizations are matched across the different hospital systems against those in the GNRMDRO registry. If a patient has a history of GNRMDRO, the most recent such culture is used for an e-mail alert that is sent to the IP at the admitting hospital.

\section{Automated E-mail Alerts}

Mirth software (Mirth Corporation) was used to handle the final steps that transform the REICON-enhanced data into e-mail documents. Secure (encrypted and with username/ password) e-mail accounts were established for the IPs. At the request of the IPs, we included, in the e-mail's subject line, an abbreviation for which rule applies: (1) ESBL-E, (2) CRE, (3) Pseudomonas, (4) Acinetobacter, or (5) other. Not all of the hospital systems are interested in all 5 rules. E-mails were tested among the project team beginning on October 29,2013 , and actual alerts were sent to the IPs at the original 5 INPC hospital systems beginning on January 29, 2014. In "Results," we describe the patients with GNRMDRO iden- tified from October 1, 2013, through December 31, 2014, and their subsequent encounters at the original 5 hospital systems through February 6, 2014. Encounter records in the database were used for this analysis.

\section{RESULTS}

\section{Analysis of Encounter Records}

From October 1, 2013, through December 31, 2013, there were 80,180 inpatients, ED patients, and outpatients of all ages with a microbiology culture parsed by the REICON system. Of those, $573(0.7 \%)$ of had a culture with GNRMDRO. Among the 573, $252(44 \%)$ had an ED or inpatient encounter after the GNRMDRO culture. Demographics, mortality, and length of follow-up of the overall cohort and of the subset of 573 are described in Table 2. In Table 3, the 573 patients with a GNRMDRO are reported in 3 subgroups: (1) those with no subsequent ED or inpatient visit, (2) those with a subsequent ED or inpatient visit in any of the 12 hospital systems, and (3) those within subgroup 2 whose subsequent ED or inpatient visit was in the original 5 hospital systems.

The 573 patients had a total of 626 distinct positive cultures for GNRMDRO. About three-fourths of the organisms were ESBL-E, and about two-thirds were obtained from urine cultures. The distributions of the organisms and the body sources are described in Table 4.

Forty-seven patients ( $8 \%$ of $573,19 \%$ of 252 ) had the subsequent ED or inpatient encounter at an institution different from the one where the culture was obtained. These 47 patients reflected 23 distinct pairs of culture institution and return encounter institution.

The results in Tables 2, 3, and 4 reflect a retrospective period (October 1, 2013, through February 6, 2014) in which we tested various aspects of the new system. The encounter 
TABLE 2. Description of the Cohort

\begin{tabular}{|c|c|c|}
\hline & $\begin{array}{l}\text { Patients with at least } 1 \\
\text { microbiology culture } \\
\text { parsed by REICON }\end{array}$ & $\begin{array}{l}\text { Patients with a culture positive } \\
\text { for a GNRMDRO (a subset } \\
\text { of the column at left) }\end{array}$ \\
\hline Total no. & 80,180 & 573 \\
\hline \multicolumn{3}{|l|}{ Age, years } \\
\hline Mean & 44 & 61 \\
\hline Standard deviation & 26 & 22 \\
\hline Minimum & 0 & 0 \\
\hline 25th percentile & 22 & 48 \\
\hline Median & 43 & 66 \\
\hline 75th percentile & 66 & 78 \\
\hline Maximum & $\geq 90$ & $\geq 90$ \\
\hline \multicolumn{3}{|l|}{ Sex } \\
\hline Female & $53,206(66)$ & $390(68)$ \\
\hline \multicolumn{3}{|l|}{ Race } \\
\hline Black & $10,670(13)$ & $44(8)$ \\
\hline Other & $7,550(9)$ & $51(9)$ \\
\hline White & $57,996(72)$ & $445(78)$ \\
\hline Missing & $3,964(5)$ & $33(6)$ \\
\hline Mortality & $541(0.7)$ & $16(2.8)$ \\
\hline \multicolumn{3}{|c|}{ Time of follow-up, days } \\
\hline Mean & NA & 89 \\
\hline Standard deviation & NA & 26 \\
\hline Minimum & NA & 0 \\
\hline 25th percentile & NA & 70 \\
\hline Median & NA & 90 \\
\hline 75th percentile & NA & 112 \\
\hline Maximum & NA & $143^{\mathrm{b}}$ \\
\hline
\end{tabular}

NoTE. Data are no. (\%), unless otherwise indicated. GNRMDRO, gram-negative multidrug-resistant organism; NA, not applicable; REICON, Regional Electronic Infection Control Network.

a Number of days from first GNRMDRO culture to February 6, 2014 (or to death if death was before February 6, 2014).

b Maximum is greater than the number of calendar days from October 1, 2013, to February 6,2014 , because a few culture results from September 2013 were sent by hospital laboratories to the Indiana Network for Patient Care during October 2013.

data (Table 3, right column) show that e-mail alerts would have been generated for 177 patients at the original 5 hospital systems during that time frame.

\section{Analysis of ADT-Driven E-mail Alerts}

The actual e-mail alerts to the IPs (January 29, 2014, through March 19,2014$)$ at the 5 original hospital systems were also assessed. During the 7 weeks, all 5 hospital systems were alerted for 67 distinct patients (minimum of 4 and maximum of 24 patients per hospital system). Nineteen $(28 \%)$ of the 67 patients were admitted to (and had the alert sent to) an institution different from the one where the culture was obtained. Those 19 alerts reflected 15 unique pairs of culture institution and return encounter institution.

The internal testing generated some additional insights. First, some ADT messages were generated for hospitalizations in the past. We considered requiring that the admission date be no more than 2 days in the past, but the IPs found that late alerts for a particular hospital/ED encounter could still help them flag relevant patients whose status was unknown to the institution.

In addition, $2 \%$ of the alerts were internally inconsistent. One alert with subject line "ESBL-E" listed the organism as "gram-positive cocci," and one alert with subject line "other gram-negative bacterium" listed a GNR but showed many of the antibiotic susceptibilities as sensitive. We found that REICON had read an original HL7 message correctly, but the sending hospital had subsequently sent another HL7 message that contradicted the first. Both cases were cultures with more than 1 organism, and the hospital sent preliminary culture findings sometime after it had sent the final culture results. The out-of-sequence message overwrote some data of interest. We therefore wrote a program in our Mirth channel to check the e-mail documents and to stop the e-mail from being sent if internally inconsistent.

A third insight involves the complexities not only of an 
TABLE 3. Description of the Cohort $(N=573)$ with a Gram-Negative Multidrug-Resistant Organism (GNRMDRO)

\begin{tabular}{|c|c|c|c|}
\hline & $\begin{array}{l}\text { Patients with no } \\
\text { subsequent } \mathrm{ED} \text { or } \\
\text { inpatient admission }\end{array}$ & $\begin{array}{l}\text { Patients with a subsequent ED } \\
\text { or inpatient admission in any } \\
\text { of the } 12 \text { hospital systems }\end{array}$ & $\begin{array}{l}\text { Patients with a subsequent ED } \\
\text { or inpatient admission in } \\
\text { the } 5 \text { hospital systems }\end{array}$ \\
\hline Total no. & 321 & 252 & 177 \\
\hline \multicolumn{4}{|l|}{ Age, years } \\
\hline Mean & 62 & 61 & 60 \\
\hline Standard deviation & 22 & 20 & 21 \\
\hline Minimum & 0 & 0 & 0 \\
\hline 25th percentile & 44 & 52 & 50 \\
\hline Median & 67 & 66 & 62 \\
\hline 75th percentile & 79 & 77 & 75 \\
\hline Maximum & $\geq 90$ & $\geq 90$ & $\geq 90$ \\
\hline \multicolumn{4}{|l|}{ Sex } \\
\hline Female & $216(67)$ & $167(66)$ & $113(64)$ \\
\hline \multicolumn{4}{|l|}{ Race } \\
\hline Black & $16(5)$ & $28(11)$ & $24(14)$ \\
\hline Other & $22(7)$ & $29(12)$ & $23(13)$ \\
\hline White & $262(82)$ & $183(73)$ & $130(73)$ \\
\hline Missing & $21(7)$ & $12(5)$ & $0(0)$ \\
\hline Mortality & $11(3)$ & $5(2)$ & $3(2)$ \\
\hline \multicolumn{4}{|c|}{$\begin{array}{l}\text { Time from first GNRMDRO culture to } \\
\text { first subsequent admission (ED or } \\
\text { inpatient), days }\end{array}$} \\
\hline Mean & NA & 30.8 & 30.0 \\
\hline Standard deviation & NA & 27.1 & 27.6 \\
\hline Minimum & NA & 1 & 1 \\
\hline 25th percentile & NA & 10 & 7 \\
\hline Median & NA & 24 & 24 \\
\hline 75th percentile & NA & 47 & 45 \\
\hline Maximum & NA & 115 & 115 \\
\hline \multicolumn{4}{|l|}{ Charlson score } \\
\hline Mean & 0.8 & 1.9 & 2.3 \\
\hline Standard deviation & 1.5 & 2.5 & 2.7 \\
\hline Minimum & 0 & 0 & 0 \\
\hline Maximum & 12 & 13 & 13 \\
\hline Median & 0 & 1 & 2 \\
\hline
\end{tabular}

NOTE. Data are no. (\%), unless otherwise indicated. ED, emergency department; NA, not applicable.

HIE itself but also of the electronic medical record (EMR) systems and data flows within multihospital systems that send data to an HIE. In recent years, large hospital systems have purchased smaller hospitals and are implementing new EMR software across multiple sites at the same time that "legacy" data feeds already connect some of the same local hospitals to the HIE. REICON sent an alert to an IP on the basis of an admission to one such local hospital. The hospital was sending data to the HIE, but the hospital's EMR was not yet connected to its own hospital system's EMR. Therefore, when the IP received the REICON alert, she could not look up the patient in her own workplace's EMR.

We monitored the number of GNRMDRO cultures per institution as well as the number of test versus actual alerts per hospital. In this way, we observed situations in which a particular hospital was not having its microbiology data processed by REICON or seemed to have too few GNRMDROs or too few e-mail alerts based on patient volume. This monitoring allowed us to revise the REICON processes for particular institutions or to investigate HIE processes upstream from REICON.

\section{DISCUSSION}

The early results suggest that this new system to deliver alerts has the potential to reduce the time to isolation and the spread of GNRMDROs. Efficient tracking of regional rates and patterns of infection may facilitate cooperation and coordination between hospitals to reduce the spread of an organism while rates are still relatively low. ${ }^{24}$ Even after initial success in limiting the spread of an MDRO, ongoing vigilance is necessary. ${ }^{25}$

In the 4-month testing period that assessed encounter records from all 12 hospital systems, at least 252 e-mail alerts 
TABLE 4. Organism Categories, Body Sources, Organism by Age, and Return Visit Types

\begin{tabular}{|c|c|c|c|c|}
\hline & $\begin{array}{l}\text { Patients with a } \\
\text { culture positive for } \\
\text { GNRMDRO }\end{array}$ & $\begin{array}{l}\text { Patients with a culture } \\
\text { positive for GNRMDRO } \\
\text { and a subsequent } \\
\text { ED or inpatient } \\
\text { admission }\end{array}$ & $\begin{array}{l}\text { Age among those in } \\
\text { the } N=252 \text { column } \\
\text { by GNRMDRO category, } \\
25 \text { th, } 50 \text { th, } 75 \text { th } \\
\text { percentile }\end{array}$ & $\begin{array}{c}\text { Care setting of first } \\
\text { return encounter } \\
\text { (ED or inpatient), } \\
\% \text { inpatient }\end{array}$ \\
\hline Total no. & 573 & 252 & 252 & 252 \\
\hline \multicolumn{5}{|l|}{ GNRMDRO category ${ }^{a}$} \\
\hline ESBL-E & $447(78)$ & $184(73)$ & $53,68,80$ & 73 \\
\hline CRE & $41(7)$ & $22(9)$ & $56,64,73$ & 86 \\
\hline Pseudomonas aeruginosa & $50(9)$ & $29(12)$ & $28,58,67$ & 93 \\
\hline Acinetobacter baumannii & $19(3)$ & $10(4)$ & $46,56,72$ & 90 \\
\hline Other' & $16(3)$ & $7(3)$ & $26,31,60$ & 100 \\
\hline \multicolumn{5}{|l|}{ Body source of culture } \\
\hline Total distinct cultures & 626 & 282 & .. & ... \\
\hline Urine & $424(68)$ & $183(65)$ & $\ldots$ & $\ldots$ \\
\hline Sputum/trachea/BAL & $80(13)$ & $38(13)$ & $\ldots$ & $\ldots$ \\
\hline Skin/wound & $40(6)$ & $22(8)$ & $\ldots$ & $\ldots$ \\
\hline Blood & $39(6)$ & $16(6)$ & $\ldots$ & $\ldots$ \\
\hline Other/nonspecific & $43(7)$ & $23(8)$ & $\ldots$ & $\ldots$ \\
\hline
\end{tabular}

NOTE. Data are no. (\%), unless otherwise indicated. BAL, bronchoalveolar lavage; CRE, carbapenem-resistant Enterobacteriaceae; ED, emergency department; ESBL-E, extended-spectrum $\beta$-lactamase-producing Enterobacteriaceae.

${ }^{a}$ First positive culture for gram-negative rod multidrug-resistant organism (GNRMDRO) during the study period.

'Stenotrophomonas maltophilia.

would have been generated, 47 to an IP at an admitting hospital system different from the hospital system where the culture was obtained. If only the original 5 hospital systems had been involved during that testing period, e-mails would have been generated for 177 patients (9-10 per week, on average). This pattern of potential e-mail alert generation was confirmed with measurement of the actual alerts: in 7 weeks, e-mail alerts for 67 patients were sent to a total of 11 hospitals in the 5 hospital systems. For the IPs, the informational value of the alerts was highest when the culture was from an institution different from the IP's own.

The population with GNRMDROs in this study is mostly older adults (median age, 66 years). The high rate of recurrent $\mathrm{ED}$ and inpatient encounters may reflect more chronic illness or debility. When analyzed by organism (Table 4), some bacteria may be more common in younger populations. Patients with e-mail alerts due to Pseudomonas or other GNRMDROs tended to be younger (25th percentile, 26-30 years old), and many had cystic fibrosis ( $52 \%$ of the 50 patients with $P$ seudomonas and 7 of the 9 patients with other GNRMDROs).

Our calculation of Charlson scores relies on International Classification of Diseases, 9th Revision (ICD-9) diagnosis codes in the INPC; it is likely that our Charlson scores are underestimates, because the INPC does not receive complete outpatient visit or ICD-9 code data from all of the institutions. However, Charlson scores were highest among patients who had a GNRMDRO and subsequently used the healthcare system. In addition, the highest scores were seen among those patients who sought care in the original 5 healthcare systems. ICD-9 diagnosis data were generally more available from those hospital systems than from institutions that joined the INPC later.

Weaknesses of this retrospective study may reflect limitations in the REICON processes, in the data that flow into REICON, or in other data in the INPC that were used in the analyses. During the study time frame, monitoring and revising the microbiology-parsing and ADT-handling procedures allowed us to fix various deficiencies when we noticed them. It is possible that not all GNRMDROs and/or not all subsequent encounters (ED or inpatient) were identified. However, the rate at which actual alerts were generated beginning on January 29,2014, was consistent with the rate of return ED or inpatient encounters (test alerts) in the 4-month retrospective analysis that began on October 1, 2013. Because the GNRMDRO registry is only a few months old, the results reported here may underestimate the future value of the alert system.

There are a few aspects of generalizability to consider. The 27 hospitals are mostly urban and reflect hospital systems of varying size (range, 1-10 hospitals). Considering all 12 hospital systems, there are 9,341 total beds, and there were 252 patients with GNRMDRO subsequently admitted to an ED or inpatient unit within a 4-month period. As the microbiology registry data accrue, we expect the rate of alerts to increase, and these figures may serve as a starting point for the development of regional estimates. A second issue is whether REICON methods might be replicated in other locales; new sites for implementation are being considered elsewhere. GNRMDROs have a high mortality rate-as high as $50 \%$ for $\mathrm{CRE}^{26}$ or Acinetobacter bloodstream infections, and 
these infections add substantially to length of stay and hospital costs. ${ }^{1.27}$ The economic impact of GNRMDROs and the potential for interhospital coordination to reduce spread ${ }^{12-17}$ suggest that a system like REICON may have a positive return on investment if implemented regionally. A cost-effectiveness analysis of REICON and its impact on GNRMDROs will be an important next step. Extension of REICON to include alerts for gram-positive MDROs, such as VRE and MRSA, will be important to demonstrate the flexibility of its infrastructure and processes.

\section{ACKNOWLEDGMENTS}

We gratefully acknowledge Mahesh Nalkande and his team at Persistent Systems, including Sumit Gurav, Preeti Lodha, and Lakhan Pardeshi, for their creative and intensive work and many vital contributions in building the REICON system. We also thank Jennifer Stanton, MPH, of Indiana University Children's Health Services Research for her reviews of the literature and of hospital characteristics in Indiana.

Financial support. This project was funded under grant 5R18HS020014 from the Agency for Healthcare Research and Quality (AHRQ), US Department of Health and Human Services. The findings and conclusions in this document are those of the authors, who are responsible for its content, and do not necessarily represent the views of AHRQ. No statement in this report should be construed as an official position of AHRQ or of the US Department of Health and Human Services.

Potential conflicts of interest. All authors report no conflicts of interest relevant to this article. All authors submitted the ICMJE Form for Disclosure of Potential Conflicts of Interest, and the conflicts that the editors consider relevant to this article are disclosed here.

Address correspondence to Marc Rosenman, MD, Children's Health Services Research, Indiana University Department of Pediatrics, 410 West 10th Street, Suite 1000, Indianapolis, IN 46202 (mrosenma@iu.edu).

\section{REFERENCES}

1. Snitkin ES, Zelazny AM, Thomas PJ, et al. Tracking a hospital outbreak of carbapenem-resistant Klebsiella pneumoniae with whole-genome sequencing. Sci Transl Med 2012;4:148ral 16.

2. Centers for Disease Control and Prevention (CDC). New Carbapenem-Resistant Enterobacteriaceae Warrant Additional Action by Healthcare Providers. CDC Health Advisory. Updated February 14, 2013. http://www.bt.cdc.gov/HAN/han00341.asp. Accessed March 11, 2013.

3. Duffy J, Sievert D, Rebmann C, et al, Effective state-based surveillance for multidrug-resistant organisms related to health care-associated infections. Public Health Rep 2011;126:176-185.

4. Kallen AJ, Srinivasan A. Current epidemiology of multidrugresistant gram-negative bacilli in the United States. Infect Control Hosp Epidemiol 2010;31(suppl 1):S51-S54.

5. Carbonne A, Arnaud I, Maugat S, et al; MDRB Surveillance National Steering Group (BMR-Raisin). National multidrugresistant bacteria (MDRB) surveillance in France through the RAISIN network: a 9 year experience. J Antimicrob Chemother 2013;68:954-959.

6. Caini S, Hajdu A, Kurcz A, et al. Hospital-acquired infections due to multidrug-resistant organisms in Hungary, 2005-2010. Euro Surveill 2013;18:pii $=20352$.

7. Khan E, Ejaz M, Zafar A, et al. Increased isolation of ESBL producing Klebsiella pneumoniae with emergence of carbapenem resistant isolates in Pakistan: report from a tertiary care hospital. J Pak Med Assoc 2010;60:186-190.

8. Datta S, Wattal C, Goel N, et al. A ten year analysis of multidrug resistant blood stream infections caused by Escherichia coli and Klebsiella pneumoniae in a tertiary care hospital. Indian J Med Res 2012;135:907-912.

9. Laxminarayan $\mathrm{R}$, Duse A, Wattal C, et al. Antibiotic resistancethe need for global solutions. Lancet Infect Dis 2013;13:10571098.

10. Doi Y, Park YS, Rivera JI, et al. Community-associated extendedspectrum $\beta$-lactamase-producing Escherichia coli infection in the United States. Clin Infect Dis 2013;56(5):641-648.

11. Birgand G, Armand-Lefevre L, Lolom I, et al. Duration of colonization by extended-spectrum $\beta$-lactamase-producing Enterobacteriaceae after hospital discharge. Am J Infect Control 2013; 41:443-447.

12. Huang SS, Taliser RA, Song Y, et al. Quantifying interhospital patient sharing as a mechanism for infectious disease spread. Infect Control Hospital Epidemiol 2010;31(11):1160-1169.

13. Lee BY, McGlone SM, Wong KF, et al. Modeling the spread of methicillin-resistant Staphylococcus aureus (MRSA) outbreaks throughout the hospitals in Orange County, California. Infect Control Hosp Epidemiol 2011;32(6):562-572.

14. Lee BY, Bartsch SM, Wong KF, et al. Simulation shows hospitals that cooperate on infection control obtain better results than hospitals acting alone. Health Aff (Milwood) 2012;31:2295-2303.

15. Lowe $\mathrm{C}$, Katz K, McGeer A. Disparity in infection control practices for multi-drug resistant Enterobacteriaceae. Am I Infect Control 2012;40:836-839.

16. Kho $\mathrm{AN}$, Lemmon $\mathrm{L}$, Commiskey $\mathrm{M}$, et al. Use of a regional health information exchange to detect crossover of patients with MRSA between urban hospitals. I Am Med Inform Assoc 2008; $15(2): 212-216$.

17. Kho AN, Doebbeling BN, Cashy JP, et al. A regional informatics platform for coordinated antibiotic-resistant infection tracking, alerting, and prevention. Clin Infect Dis 2013;57:254-262.

18. Rosenman MB, Szucs KA, Finnell SME, et al. Turning unstructured microbiology culture data into usable information: methods for alerting infection preventionists in a health information exchange about multidrug-resistant gram-negative bacterial infections. In: Battles JB, Cleeman JI, Kahn KK, Weinberg DA, eds. Advances in the Prevention and Control of HealthcareAssociated Infections. Rockville, MD: Agency for Healthcare Research and Quality, 2014.

19. McDonald CJ, Overhage JM, Barnes M, et al. The Indiana Network for Patient Care: a working local health information infrastructure. Health Aff 2005;24(5):1214-1220.

20. Sittig DF, Hazlehurst BL, Brown J, et al. A survey of informatics platforms that enable distributed comparative effectiveness research using multi-institutional heterogenous clinical data. Med Care 2012;50(suppl):S49-S59.

21. Kho AN, Dexter P, Lemmon L, et al. Connecting the dots: creation of an electronic regional infection control network. Stud Health Technol Inform 2007;129(pt 1):213-217.

22. Kho AN, Lemmon L, Dexter PR, et al. An operational citywide electronic infection control network: results from the first year. AMIA Annu Symp Proc 2008; Nov 6:1222.

23. Health Level Seven International. http://www.hl7.org/. Accessed May 31, 2014. 
24. Smith DL, Levin SA, Laxminarayan R. Strategic interactions in multi-institutional epidemics of antibiotic resistance. Proc Natl Acad Sci USA 2005;102:3154-3158.

25. Cooper BS, Medley GF, Stone SP, et al. Methicillin-resistant Staphylococcus aureus in hospitals and the community: stealth dynamics and control catastrophes. Proc Natl Acad Sci USA 2004; 101:10223-10228.
26. Patel G, Huprikar S, Factor SH, et al. Outcomes of carbapenemresistant Klebsiella pneumoniae infection and the impact of antimicrobial and adjunctive therapies. Infect Control Hosp Epidemiol 2008;29:1099-1106.

27. Lee N-Y, Lee H-C, Ko N-Y, et al. Clinical and economic impact of multidrug resistance in nosocomial Acinetobacter baumannii bacteremia. Infect Control Hosp Epidemiol 2007;28:713-719. 